\title{
89
}

\section{REGULATION DE L'EXPRESSION DU PROTO-ONCOGENE C-FOS PAR L'HORMONE SOMATOTROPE AU COURS DE LA DIFFERENCIATION DES PREADIPOCYTES EN CULTURE}

Barcellini-Couget, S., Doylio A., Cirimaldi P., Dani C.

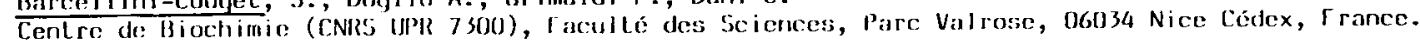

Le traitement à long terme des cellules préadipocytaires par l'hormone somatotrope (GH) est nécessaire à l'expression des gènes de différenciation terminale (GPDH, aP2, adipsine, ...) sans que les mécanisınes d'action de l'hormone ne soient ćlucidés. Outre cet effet à long terme de la GH sur les préadipocytes, des événements beaucoup plus rapides de l'hormone peuvent être mis en évidence : ainsi la transcription du proto-oncogène c-fos est activée 15 minutes après l'ajout de GH à des cellules confluentes maintenues en sérum. Comme pour d'autres inducteurs connus du gene fos (la PGF $2 \alpha$ et les facteurs de croissance), l'effet est transitoire, implique la formation de diacylglycérol (DAG), l'activation de la protéine kinase $C$ (PKC) et ne nécessite pas de synthèse protéique. Cependant l'activation du gène fos par la GH semble mettre en jeu une voie métabolique dont le précurseur de DAG n'est pas le phosphatidyl-inositol. De plus l'inhibition de la synthèse protéique ne permet pas de prolonger la réponse à l'hormone comme cela a été décrit pour les autres inducteurs. Ces résultats suggèrent fortement que la régulation du gène c-fos par la GH dans les cellules adipocytaires met en jeu une voie d'activation et/ou des éléments regulateurs sur le gene différents de ceux caractérisés à ce jour.

Le proto-oncogène $c$-fos code pour une protéine nucléaire qui jouerait, en coopération avec d'autres facteurs transcriptionnels, un rôle dans la régulation positive ou négative de gènes en réponse à des signaux extra-cellulaires. Récemment il a été mis en ćvidence dans le laboratoire de B. Spiegelman (Boston, E.U.) que la protéine Fos intervient in vitro dans la formation du complexe nucléoprotéique se fixant à l'élément régulateur FSE ("fat cell-specific element") du gène aP2 induit au cours de la différenciation adipocytaire. Ainsi l'induction du gène c-fos par la GH dans les cellules en voie de différenciation pourrait être l'un des relais dans le mécanisme d'action de la GH conduisant à l'induction des gènes tardifs de conversion adipocytaire.

\section{0}

UNE PRODUCTION ACCRUE DE FACTEURS DE CROISSANCE ET UN HAUT NIVEAU D'AMPLIFICATION DU GENE C-MYS DIFTERENCIE I CS CLONES TUMORIGENES DES CLONES NON TUMORIGENES DE LA LUGEL: DE CANCER DU SEIN SW613-S

Brison O ${ }^{1}$ Lamonerie T. ${ }^{1}$, Modjtahedi N. ${ }^{1}$, Binoux M. ${ }^{2}$, Hossenlopp P. $^{2}$, Haddada H. ${ }^{3}$, Lazar E ${ }^{3}$

1) CNRS URA 1158, IGR, Villejuif ; 2) INSERM U 142, Hopital Trousseau, Paris ; 3) CNRS UPR 275, IRSC, Villejuif, FRANCE.

Le gène c-myc est amplifié dans la lignée SW613-S qui a été établie à partir d'un carcinome mammaire humain. Une analyse de clones cellulaires a montré que cette lignée est hétérogène aussi bien du point de vue du nombre de copies du gène c-myc par cellule que de leur localisation chromosomique. En effet ces copies sont soit portées par des chromosomes minuscules doubles soit intégrées dans un chromosome. Les clones ayant un haut niveau d'amplification de c-myc sont tumorigènes chez la souris athymique alors que ceux ayant un niveau faible ne le sont pas. L'introduction de copies du gene c-myc par transfection dans les cellules de plusieurs clones non tumorigenes leur confere un phénotype tumorigene ce qui indique qu'un haut niveau d'amplification de c-myc contribue à la tumorigénicité de ces cellules chez l'animal.

La croissance des clones non tumorigènes in vitro en milieu chimiquement défini et sans sêrum est beaucoup plus limitée que celle des clones tumorigenes, ce qui suggère que ces deux types cellulaires ont des exigences en facteurs de croissance différentes. Une étude comparée de milieux conditionnés pour la présence de TGF- $\alpha$ et d'IGFII a montré que ces deux facteurs de croissance sont surproduits par les clones ayant un haut niveau d'amplification de c-myc. Des expériences de transfert northern et de cartographie à la RNase ont confirmé que ces cellules renfermaient une plus grande quantité des mRNA du TGF- $\alpha$ et de l'IGFII que les cellules ayant un petit nombre de copies de c-myc. Des transferts western sondés avec de l'IGF marqué à l'iode 125 ont montré que la surexpression d'IGFII était accompagnée d'une sectétion accrue des protéines porteuses d'IGF. Des expériences préliminaires indiquent que le gène du PDGF-A est aussi exprimé différentiellement. Nos résultats montrent que les clones tumorigènes de SW613-S surexpriment plusieurs gènes impliqués dans le controlic de la proliferation cellulaire ce qui pourrait expliquer, au moins en partie, leur plus grande capacité a prolifêrer dans un milieu sans sérum. Des expériences sont en cours pour déterminer s'il y a une relation entre la production de facteurs de croissance et l'amplification du gène c-myc dans ces cellules. 